Research Article

\title{
Structural Parameters Optimization of Corona Ring of Tethered Balloon-Type VLF Antenna
}

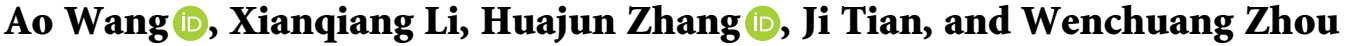 \\ School of Automation, Wuhan University of Technology, Wuhan 430070, China \\ Correspondence should be addressed to Huajun Zhang; zhanghj@whut.edu.cn
}

Received 25 February 2021; Revised 25 June 2021; Accepted 5 July 2021; Published 14 July 2021

Academic Editor: Chien-Jen Wang

Copyright (c) 2021 Ao Wang et al. This is an open access article distributed under the Creative Commons Attribution License, which permits unrestricted use, distribution, and reproduction in any medium, provided the original work is properly cited.

\begin{abstract}
The antenna end of the tethered balloon-type high-power VLF communication system will be exposed to an extremely strong alternating electric field environment when it is in operation. Due to dielectric loss, an abnormal temperature rise may occur at the antenna end, which may lead to an antenna fracture in serious cases. It is an effective measure to install a corona ring at the end of the antenna to prevent such accidents. In this paper, the structural parameters of the corona ring, including ring radius, tube radius, and ring depth, are optimized based on intelligent optimization algorithm to significantly improve the electric field distribution and restrain the temperature rise to the maximum extent. The effectiveness of the optimization results is verified by simulation.
\end{abstract}

\section{Introduction}

The very low-frequency (VLF) communication system has the advantages of long transmissible distance, good penetrating characteristics, strong anti-interference abilities, and high reliability. At present, there are two main types of VLF communication systems: stationary type and mobile type. Due to its superior mobility and concealment, the mobile type VLF communication system, whose antenna is lifted by a tethered balloon, has been paid more attention and has been widely used in military and civil fields. However, when the tethered balloon-type high-power VLF communication system transmits signals, the antenna end will be exposed to a very strong alternating electromagnetic environment. A considerable temperature rise will occur at the end of the antenna due to dielectric loss. In several cases, the antenna may be damaged or even broken due to excessive temperature. Especially for the tethered balloontype high-power VLF communication system, the antenna rupture will cause the disaster of the mooring platform disconnecting [1]. Therefore, the distribution characteristics of the electric field at the antenna end of the tethered balloon-type VLF communication system deserve more attention.
It is an effective measure to install a corona ring at the end of the antenna to improve the electric field distribution and restrain temperature rise [2]. The structure parameters of the corona ring, including ring radius, tube radius, and ring depth, directly affect the electric field distribution improvement. In this paper, based on the numerical analysis of the electric field distribution at the antenna end and the influence mechanism of the corona ring structural parameters on the electric field, the corona ring structural parameters are optimized by using intelligent algorithm so as to improve the electric field distribution at the antenna end to the greatest extent and avoid the catastrophic accident of the antenna fracture.

The articles introducing the VLF communication system are abundant. Turner has introduced the submarine communication systems, which consist of the VLF, HF, and UHF antenna systems [3]. The three configurations of the immersed receiving antennas are investigated through the theoretical, numerical, and experimental methods by Benhabiles et al. The first configuration possesses small horizontal wire sections, while the second and the third have horizontal sections of length $L$ and $2 L$, respectively. The conclusion is that the more horizontal wire parts that are between the plates, the higher the signal level [4]. By finite 
element method (FEM) simulation, Chen et al. [5] have analyzed the two types of the VLF antenna on aircraft including the single and the dual trailing wire antenna, which are used to transmit messages to submarines. The simulation result shows that the dual trailing antenna has smaller input impedance and higher input efficiency of the transmitter compared to the single one. Therefore, the airborne VLF dual trailing antenna system is better. For further improvement of the input efficiency of airborne VLF dual trailing, the solution that establishes good match between the transmitter and the antenna is suggested. Hurdsman et al. [6] have built the navy VLF transmit antenna supported by the tall tower based on the facilities of the naval station using the method of moments wire modeling code, Expert MININEC Broadcast Professional. The accuracy of the model is tested by comparing the measured and the modeled values of self-resonance frequency and radiation resistance. Liang et al. [7] have built the model of VLF valley transmitting antenna according to the method of moments. Through comparing the calculated and measured values of input reactance, the model is proved to be effective. Koons et al. have described the transmitter facility and the tethered balloon antenna that was used in Alaska. Through the measurement of electric field intensity as a function of distance from balloon lofted antenna and radiated power by antenna as a function of frequency, the performance of the facility is worth relying [8]. With the help of the method of moments and the butterfly mating optimization, $\mathrm{Li}$ et al. [9] have minimized electric field of top-wire insulator grading rings on the VLF transmitting antenna.

Several research studies have focused on the structure optimization of the grading ring in the power system. Based on the computation of the electric field intensity by FEM software, the particle swarm optimization (PSO) approach with a dynamic population size has been used by M'hamdi et al [2] to reach the optimal design of the corona ring on an HV composite insulator. Zhang et al. [10] have investigated the corona discharges by means of ultraviolet imager observation which indicates improper corona ring structure and implemented PSO algorithm to optimize the corona ring structure for ultrahigh-voltage composite insulator using APDL language in ANSYS computing environment. To avoid serious damages due to the arrester failure caused by outside or inside breakdown, Aghaebrahimi et al. [11] have applied the differential evolution and PSO to optimize the parameters of the grading ring to make the electric field on oxide surge arresters uniform. Through the simulations and laboratory tests, Ilhan et al. [12] have determined the optimal dimensions of the R-type and C-type corona rings for a $380 \mathrm{kV}$ insulator string after the comprehensive consideration of the radio interference voltage levels, ac flashover levels, arc distance, maximum electric field, and economic points. The Nelder-Mead method, bound optimization by quadratic approximation, and constrained optimization by linear approximation have been used to determine the optimal design for a corona ring [13].

From what has been discussed above, research studies on VLF communication antennas are mostly focused on their efficiency and performance. In the research of corona ring, though the investigations mentioned above have focused on the performance of the corona ring in restraining the electric field distortion, the purpose of the optimization is to inhibit the corona inception so as to ensure the safety of instruments. The optimal structure of the corona ring to solve the overheating problem at the terminal of the VLF antenna has seldom been reported.

In this paper, the three-dimensional model of the VLF antenna and the corona rings with various parameters including tube radius, ring radius, and ring depth are described in Section 2. Based on the simulation results, the nonlinear mathematical object function which implied the relationship between the maximum electric field and the dimensions is introduced in Section 3. Section 4 describes the procedure of optimizing the structure parameters of the corona ring through the PSO and simulated annealing (SA) algorithms. At last, the results obtained by optimization are verified via simulation in Section 5 .

\section{Theoretical Analysis and Simulation Model}

\subsection{Theoretical Analysis of Electric Field and Dielectric Loss.} When the high-power VLF communication system transmits signals, the voltage at the end of the antenna is very high, and correspondingly, the electric field intensity at the end of the antenna is also very high which could be up to $10^{7} \mathrm{~V} / \mathrm{m}$. However, according to the antenna theory, the current at the end of the antenna is very low which almost approximates to the zero value. The frequency of the VLF is $3-30 \mathrm{kHz}$ which is much higher than the industrial one [14]. When analyzing the electric field distribution at the end of VLF antenna, it can be considered as an electro-quasistatic field. In other words, the induced electric field is so low compared to the coulomb field that could be neglected within Maxwell's equation and shall not cause too much error. The equations are as follows:

$$
\left\{\begin{array}{l}
\nabla \times \vec{E} \approx 0 \\
\nabla \cdot \vec{D}=\rho \\
\nabla \times \vec{H}=\gamma \vec{E}+j \omega \varepsilon \vec{E} \\
\nabla \cdot \vec{B}=0
\end{array}\right.
$$

According to (1), the quasistatic field could be expressed by Poisson's equations of electric potential as follows:

$$
\left\{\begin{array}{l}
\vec{E}=-\nabla \varphi, \\
\nabla^{2} \varphi=-\frac{\rho}{\varepsilon},
\end{array}\right.
$$

where $\varphi$ represents the potential. With the dielectric loss angle $\tan \delta$ introduced and the ignorance of both the conductive and the magnetization loss because of the low current and frequency, the dielectric loss could be simplified as follows: 


$$
\begin{aligned}
\tan \delta & =\frac{\omega \varepsilon^{\prime \prime}+\gamma}{\omega \varepsilon^{\prime}}, \\
P_{l} & =\frac{\omega \varepsilon^{\prime}}{2} \tan \delta \int_{V}|\vec{E}|^{2} \mathrm{~d} v,
\end{aligned}
$$

where $P_{l}$ represents the power of dielectric loss, $\omega$ represents the angular frequency, $\varepsilon^{\prime \prime}$ represents the imaginary part of complex permittivity, and $\varepsilon^{\prime}$ represents its real part.

According to (3), the dielectric loss power is proportional to the squared electric field intensity. Therefore, it is effective to limit the dielectric loss and temperature rise by reducing the electric field strength at the terminal of the VLF antenna.

2.2. Model of Antenna and Corona Ring. In general, the tethered balloon-type VLF communication system is composed of signal transceiver, tuning system, winch system, ground system, balloon, and antenna cable. The tuning system is used to tune the antenna reactance by an inductor to match the system impedance. The winch system is responsible for handling the antenna to control the lifting height and speed. The ground system is installed to provide adequate ground contact to ensure the normal operation of the communication system [6]. The diagram of the tethered balloon-type VLF communication system is shown in Figure 1.

The model of the antenna with a corona ring is shown in Figure 2. In the actual system, the end of the VLF antenna, which is shown in the red rectangle box in Figure 2, is connected to the epoxy mandrel of the composite insulator. If the electric field intensity is too high, the temperature of the epoxy mandrel may increase due to dielectric loss, and the epoxy mandrel may break in several cases, causing incalculable losses. Therefore, the electric field intensity at the position shown in the red rectangle box in Figure 2 is the focus of this paper. The purpose of subsequent structural optimization is to minimize the electric field intensity at this location.

The antenna cable consists of aramid fiber, polyethylene inner sheath, copper layer, and semiconductive outer sheath, respectively, which is shown in Figure 2(c).

Considering the weight and the skin effects, the corona ring was designed to be hollow and made of aluminium. The structure and the parameters of the corona ring are shown in Figure 3. The connecting part is linked by a spline curve to avoid the electric field concentration at the edge. The fitting was also made of aluminium. The conductivity and relative permittivity of the materials of the antenna cable are described in Table 1.

\section{Simulation Results and the Relationship Function}

According to the actual operating parameters of a highpower VLF communication system, the AC excitation voltage and frequency were set to $120 \mathrm{kV}$ and $20 \mathrm{kHz}$ in the simulation. Depending on Maxwell's equation, the computations of the electric field were transformed to solve the

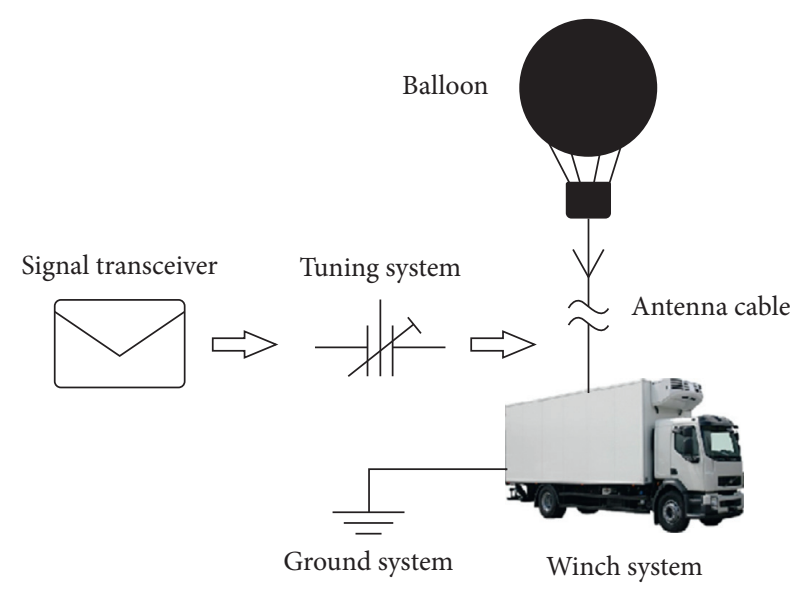

FIgURE 1: Schematic diagram of the tethered balloon-type VLF communication system.

partial differential equations. The numerical solutions were sought through employing the 3D FEM software COMSOL by solving Poisson's equation for dielectric and conducting media [2].

3.1. Effect of the Corona Ring Implementation. To figure out the influence of the ring implementation, the simulation results of models with a medium-sized ring and without corona ring were compared. The structural parameters of the medium-sized ring are as follows: the tube radius is $20 \mathrm{~mm}$, the ring radius is $225 \mathrm{~mm}$, and the ring depth is $200 \mathrm{~mm}$. The electric field distributions of the models are shown in Figures 4 and 5 . It can be seen from Figure 4 that the maximum electric field intensity at the antenna end is $4.50 \times 10^{6} \mathrm{~V} / \mathrm{m}$ when there is no corona ring. When the corona ring is added, the maximum electric field intensity at the antenna end decreases to $1.36 \times 10^{6} \mathrm{~V} / \mathrm{m}$, which decreases by about $70 \%$. Therefore, it is reasonable to conclude that applying the corona ring is an effective method to restrain electric field distortion at the terminal of the VLF antenna.

3.2. Influence of Structural Parameters of Corona Ring. To optimize the structural parameters of the corona ring, it is necessary to determine the influence mechanism of each parameter on the electric field intensity at the antenna end. When the size of corona ring is small, the effect of improving electric field intensity is limited. However, when the corona ring size is larger, its weight is larger and its vibration is greater due to the influence of wind at a high altitude. In this paper, after comprehensively considering the influence of various factors, the variation ranges of structural parameters of the corona ring to be optimized are as follows:

$$
\begin{array}{r}
10 \mathrm{~mm} \leq r \leq 40 \mathrm{~mm}, \\
150 \mathrm{~mm} \leq R \leq 300 \mathrm{~mm}, \\
100 \mathrm{~mm} \leq S \leq 300 \mathrm{~mm},
\end{array}
$$

where $r$ refers to the tube radius, $R$ is the ring radius, and $S$ is the ring depth. These parameters of the ring are shown in Figure 3 . 


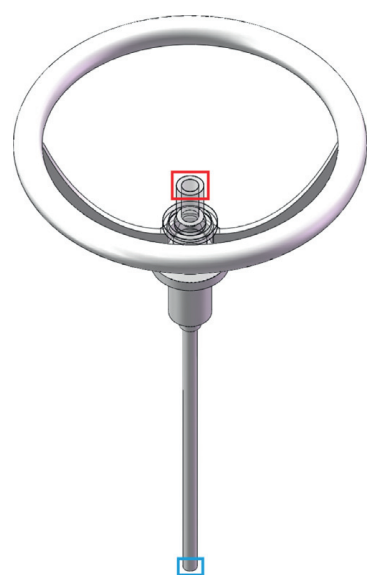

(a)

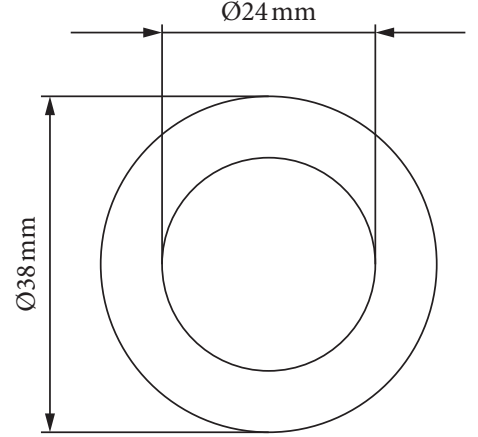

(b)

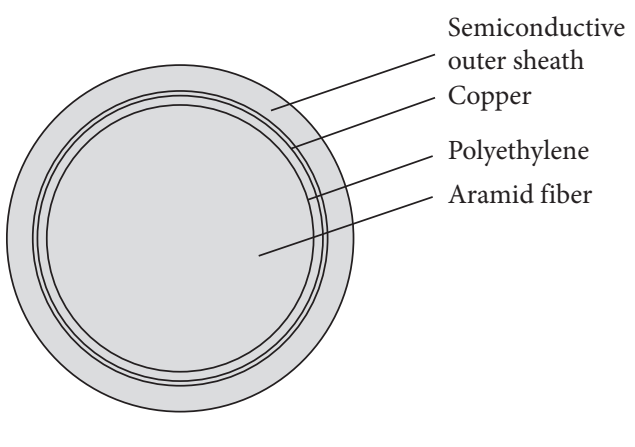

(c)

Figure 2: (a) Model of the VLF antenna with corona ring. (b) Enlargement of the cross section of the red rectangle box in (a). (c) Enlargement of cross section of the blue rectangle box in (a).

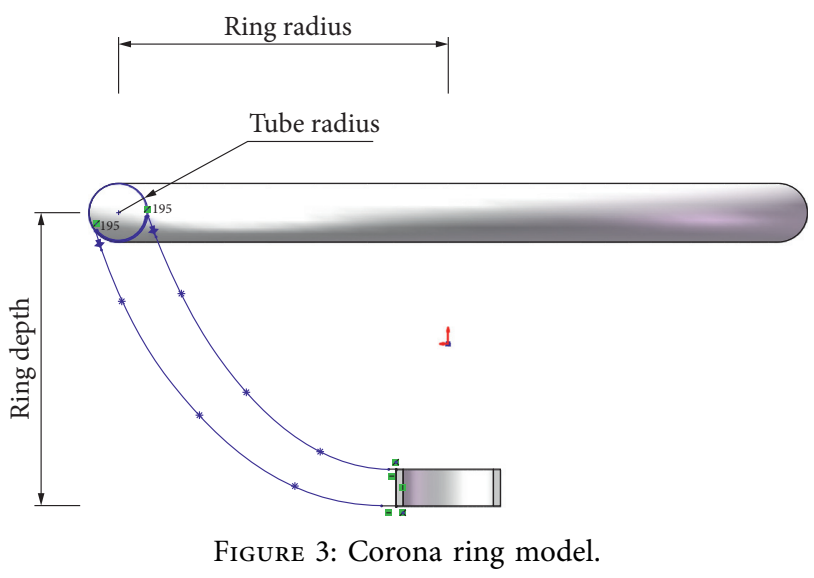

TABLE 1: Material electrical parameters.

\begin{tabular}{lcc}
\hline Material & $\begin{array}{c}\text { Relative } \\
\text { permittivity }\end{array}$ & $\begin{array}{c}\text { Conductivity } \\
\left(\mathrm{S} \cdot \mathrm{m}^{-1}\right)\end{array}$ \\
\hline Aramid fiber & 3.30 & $1.00 \times 10^{-14}$ \\
Polyethylene & 2.00 & $1.00 \times 10^{-11}$ \\
Copper & - & $5.99 \times 10^{7}$ \\
$\begin{array}{l}\text { Semiconductive outer } \\
\text { sheath }\end{array}$ & 11.4 & 1.00 \\
Aluminium & - & $3.8 \times 10^{7}$ \\
\hline
\end{tabular}

In order to figure out the effects of each parameter on reducing the maximum electric field intensity, the tube radius, ring radius, and ring depth of the corona ring were varied from their initial value to final ones by steps of $5 \mathrm{~mm}$, $25 \mathrm{~mm}$, and $25 \mathrm{~mm}$, respectively, and only one variable was changed while the two other were kept constant to gain a series of relationship curves. The curves are presented in Figures 6-8. When the curves of the relationship between the maximum electric field intensity and tube radius gets overlapped around the medium ring depth value, the lines are divided into two parts, which are bounded by the ring depth value of $200 \mathrm{~mm}$ for vividness.

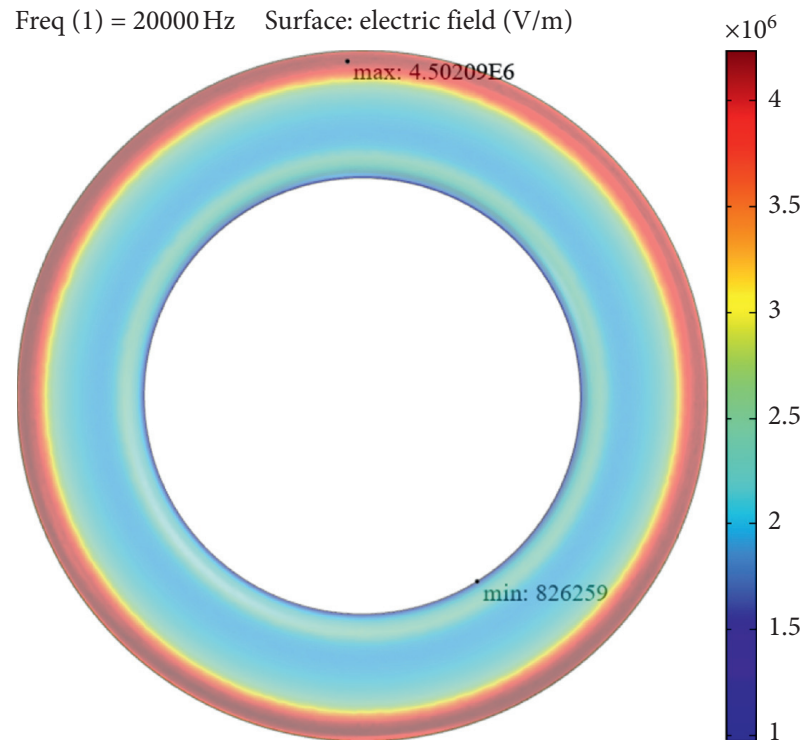

FIgURE 4: The electric field distribution at the end of the VLF antenna without corona ring.

According to the results, as the tube radius increases or as the ring radius decreases, the electric field intensity has a tendency of monotonic decrease. Moreover, as the ring depth grows, the electric field intensity appears to abate to reach a minimum value first and then rebound. Through the analysis of the numerical value, the increase in the tube radius has a remarkable effect on decreasing the electric field intensity at the antenna end. Comparatively speaking, the change in the ring radius or ring depth parameter is less effective. Therefore, for achieving the minimum electric field at the terminal, the priority is to employ the bigger tube radius value. As for other parameters, the ring radius shall be kept to the lower value. The ring depth shall be limited to an appropriate range to keep away from the values which are either too much or too little. 


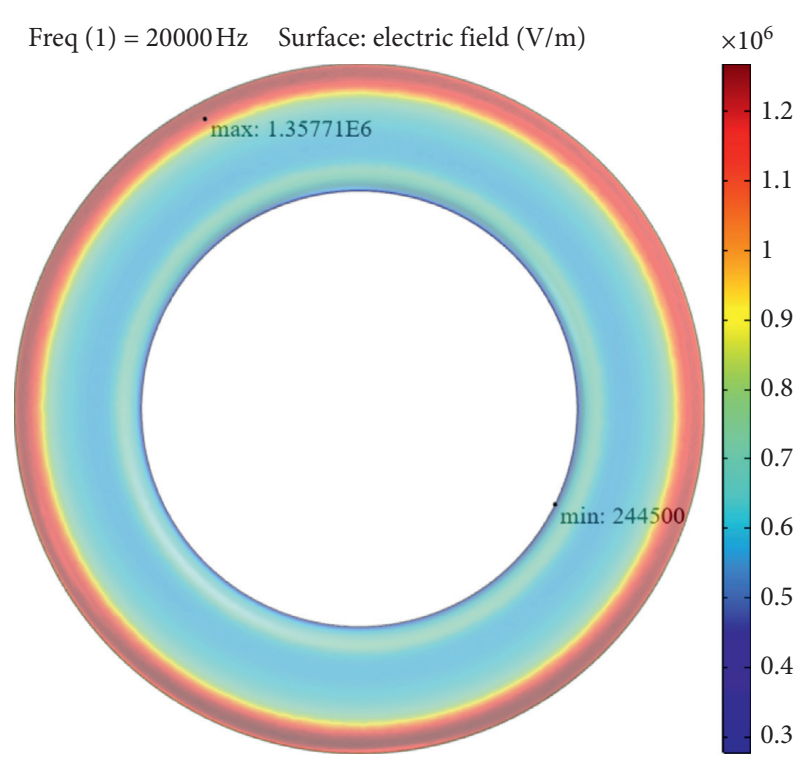

FIgURE 5: The electric field distribution at the end of the VLF antenna with the medium-sized corona ring.

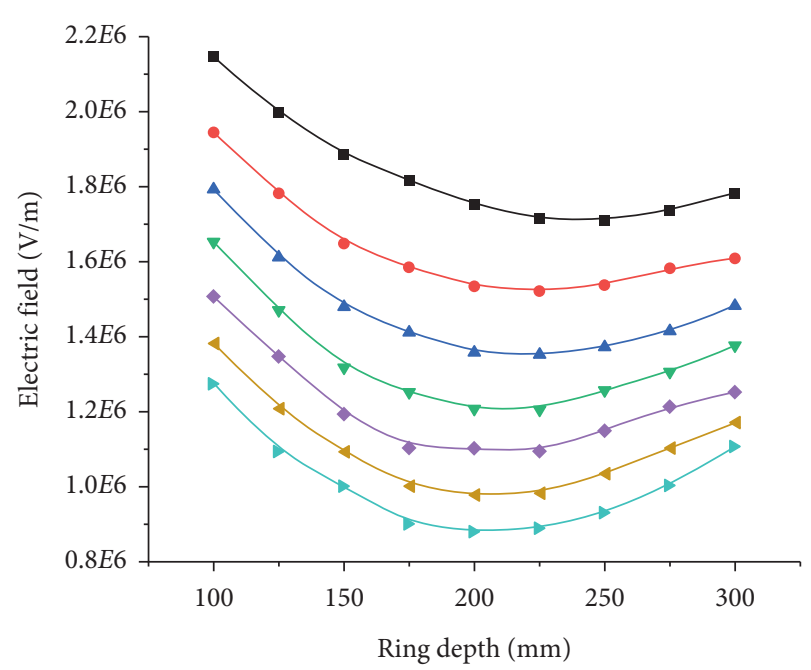

Ring tube radius

$$
\begin{array}{rlrl}
\rightarrow r & =10 \mathrm{~mm} & \rightarrow r=30 \mathrm{~mm} \\
\multimap r & =15 \mathrm{~mm} & \rightarrow r=35 \mathrm{~mm} \\
\multimap r=20 \mathrm{~mm} & \rightarrow r r=40 \mathrm{~mm} \\
\rightarrow r=25 \mathrm{~mm} & &
\end{array}
$$

Figure 6: The relationship between the maximum electric field intensity at the concerned region of the VLF antenna and the ring depth for different ring tube radii and a constant ring radius of $225 \mathrm{~mm}$.

3.3. Relationship Function. On the basis of the simulation results, the tendency between the electric field and the tube radius approximates to linear relationship. So does the ring radius with the opposite monotonicity. As for the ring depth, the curves seem to submit to a quadratic equation. For that, there are three independent variables, and traditional polynomial fitting function cannot solve such

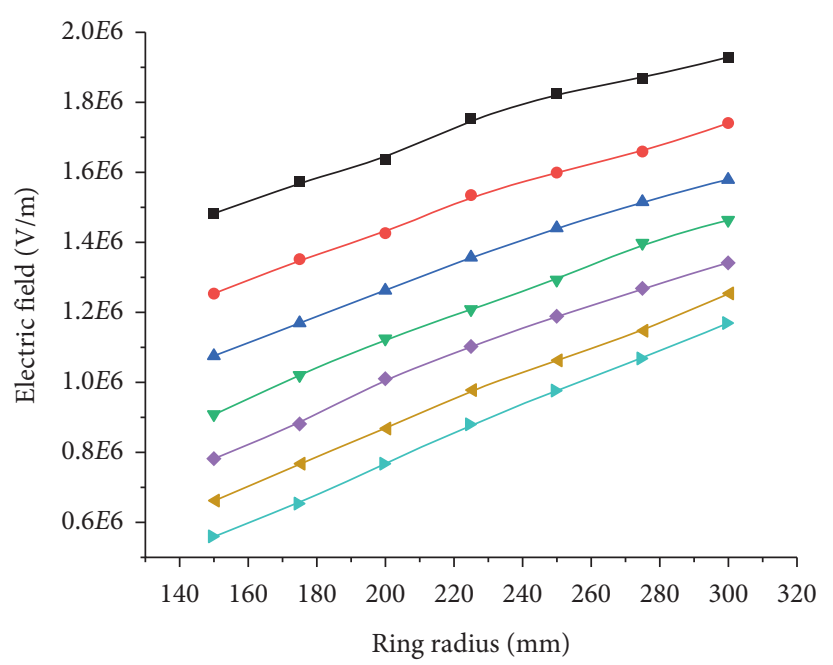

Ring tube radius

$$
\begin{aligned}
& \text { - } r=10 \mathrm{~mm} \\
& -r=30 \mathrm{~mm} \\
& \multimap r=15 \mathrm{~mm} \\
& \longleftarrow r=35 \mathrm{~mm} \\
& \neg r=20 \mathrm{~mm} \\
& \rightarrow r=40 \mathrm{~mm} \\
& \rightarrow r=25 \mathrm{~mm}
\end{aligned}
$$

FIgURE 7: The relationship between the maximum electric field intensity at the concerned region of the VLF antenna and the ring radius, for different ring tube radii and a constant ring depth of $200 \mathrm{~mm}$.

multiparameter fitting problems. Therefore, a polynomial was proposed to establish the function by the method of multivariate nonlinear regression as follows:

$$
E=\sum_{i, j, k} a_{i j k} r^{i} R^{j} S^{k} \quad i, j=0,1, k=0,1,2,
$$

where $a_{i j k}$ are the polynomial coefficients and the symbol $E$ refers to the maximum electric field intensity at the terminal of antenna.

The performance of the fitting is assessed by the coefficient of determination that is 0.9730 in the nonlinear regression result. Therefore, it is a satisfactory function to reflect the relationship function within the ranges illustrated above.

\section{Optimization of the Parameters}

Based on the relationship function, the optimization of the structure parameters was carried out. It was significant to choose the suitable algorithms with the tradeoff between simplicity and efficiency to achieve this purpose. Amongst all the stochastic search optimization methods, particle swarm optimization (PSO) and simulated annealing (SA) have accelerated gaining attention and applications by scholars and researchers because of their simple concepts, good speed, and ease of implementation [15]. PSO is one of the most efficient but simple computational methods that show a robust performance in various applications. It ensures an adequate tradeoff between complexity, accuracy, and speed convergence [16]. However, the algorithm has a 


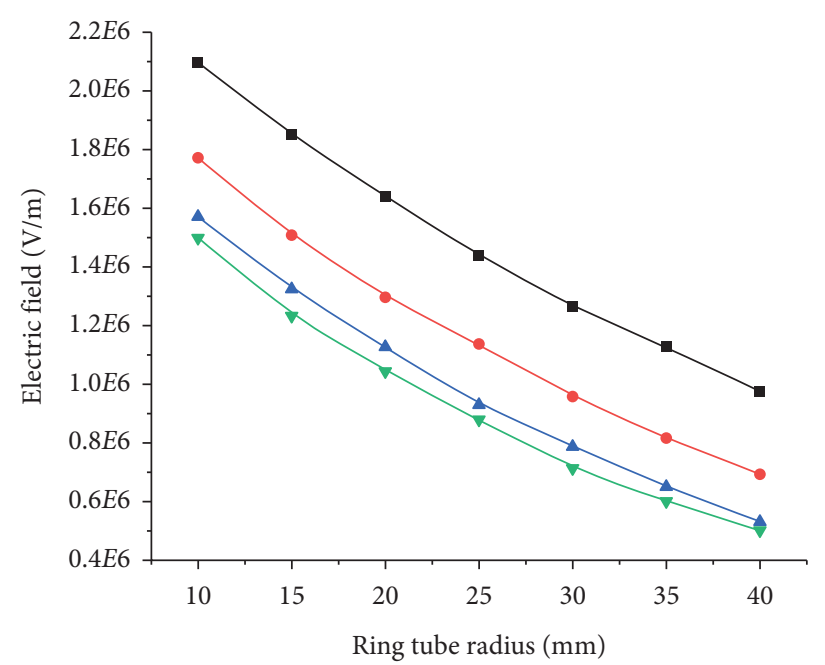

Ring depth

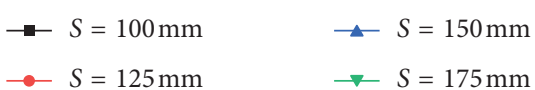

(a)

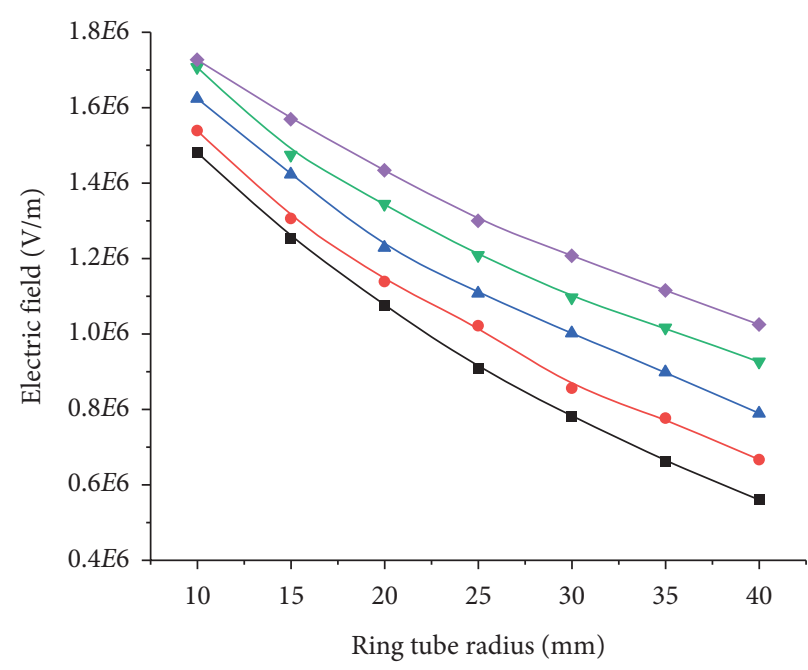

Ring depth

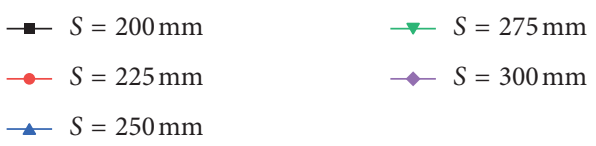

(b)

FIGURE 8: The relationship between the maximum electric field intensity at the concerned region of the VLF antenna and the ring tube radius, for different ring depths and a constant ring radius of $150 \mathrm{~mm}$.

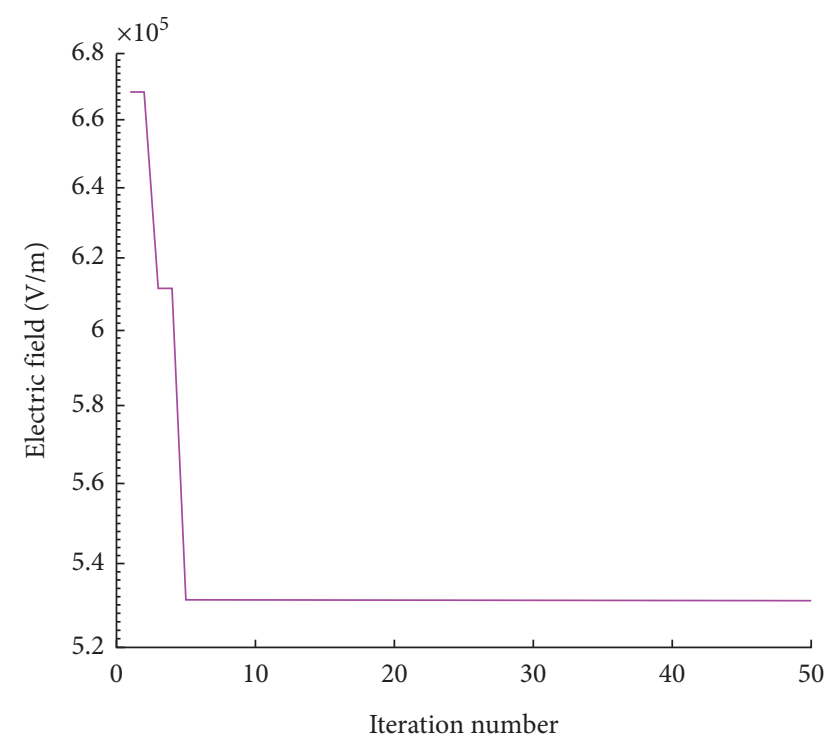

FIGURE 9: The process of the optimization through the PSO method by the toolbox.

poor exploitation which may make it fail to refine the visited area in the solution spaces [17]. In contrast to PSO, SA is known as a stable method having a mechanism to jump out of local minima [15]. To chase for the efficiency of the iterations, the PSO was used to gain the result. Then, the SA was used to confirm the accuracy of the first result to prevent the situation that the particles got stuck to the local optimal value.
4.1. Optimization through PSO. The PSO algorithm was proposed by Kennedy and Eberhart in 1995 [18]. It comprises a very simple concept, and paradigms can be implemented in a few lines of computer code [18]. The algorithm uses two vectors, the velocity and position, which are updated by using the following equation to define the particle in the search space:

$$
\begin{aligned}
V_{i}^{k+1} & =w V_{i}^{k}+c_{1} r_{1}\left(P_{i}^{k}-X_{i}^{k}\right)+c_{2} r_{2}\left(P_{g}^{k}-X_{i}^{k}\right) X_{i}^{k+1} \\
& =X_{i}^{k}+V_{i}^{k+1},
\end{aligned}
$$

where $w$ is the inertia weight to balance exploration and exploitation ability in search space, $V_{i}^{k}$ and $X_{i}^{k}$ are the velocity and position of the $k$ th particle, respectively, $P_{i}^{k}$ is the individual optimal value in the $k$ th cycle, $P_{g}^{k}$ is the global optimal value in the $k$ th cycle, $c_{1}$ and $c_{2}$ denote learning factors, and $r_{1}$ and $r_{2}$ refer to random numbers with values between the range of $(0,1)$. The first part $w V_{i}^{k}$ is part of the momentum, which is the memory capacity of particles; the second part $c_{1} r_{1}\left(P_{i}^{k}-X_{i}^{k}\right)$ is part of cognitive that reflects the update of particle's velocity; and the third part $c_{2} r_{2}\left(P_{g}-X_{i}^{k}\right)$ is the part of the society which can lead particles to fly to the best location of the swarm [19].

The PSO toolbox was used to achieve the goal of optimization. The toolbox parameters were set to the option that the maximum number of epochs was 50 , the population size was 24 , the initial and final inertia weights were 0.9 and 0.4 , respectively, the acceleration constants of the local and global best influence were taken as 2 , and the maximum velocity changing range was $20 \%$. The process of the optimization is shown in Figure 9. The particles are converged to the optimal electric field intensity value and the 
TABLE 2: Optimal electric field intensity value and the corresponding ring dimensions gained by two algorithms.

\begin{tabular}{lcc}
\hline Algorithm & Optimal electric field norm $\left(\mathrm{V} \cdot \mathrm{m}^{-1}\right)$ & Corresponding corona ring dimensions $(\mathrm{mm})$ \\
\hline PSO & 531022 & $r=40, R=150$, and $S=192.840$ \\
SA & 531022 & $r=40, R=150$, and $S=192.835$ \\
\hline
\end{tabular}

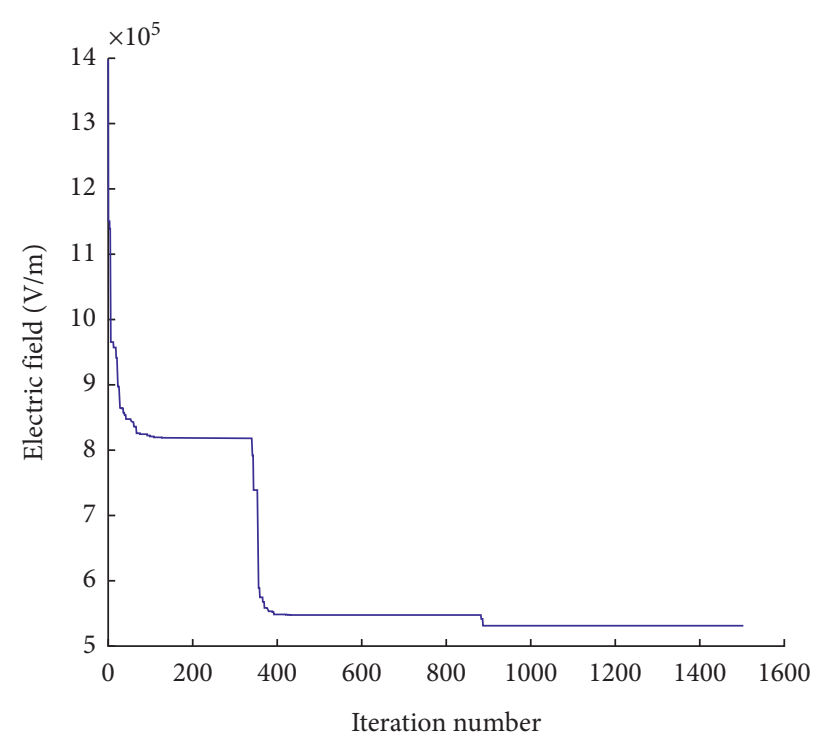

FIgURE 10: The process of the optimization through the PSO method by the toolbox.

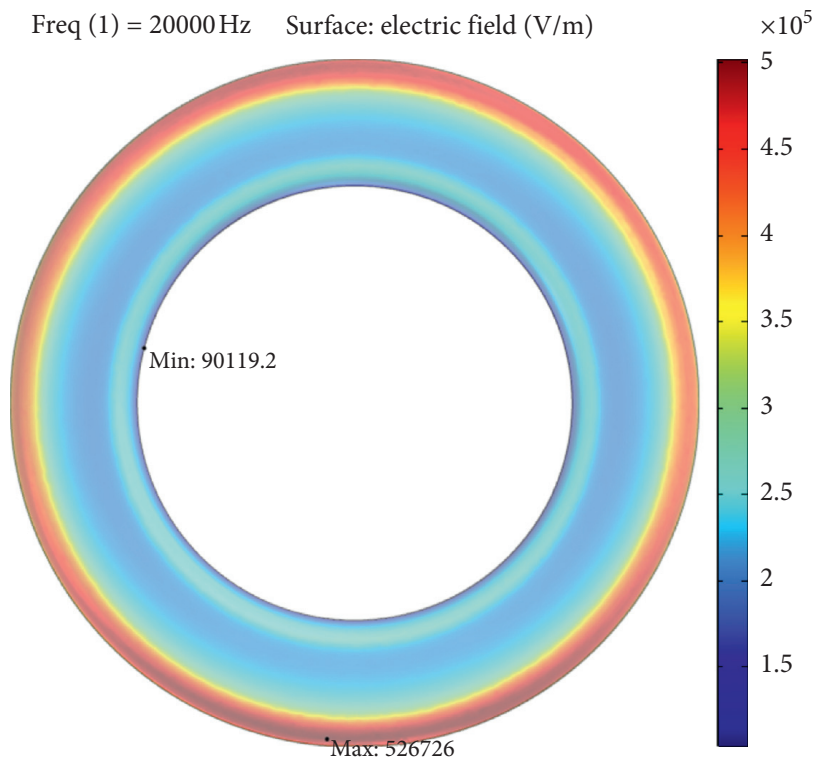

(a)

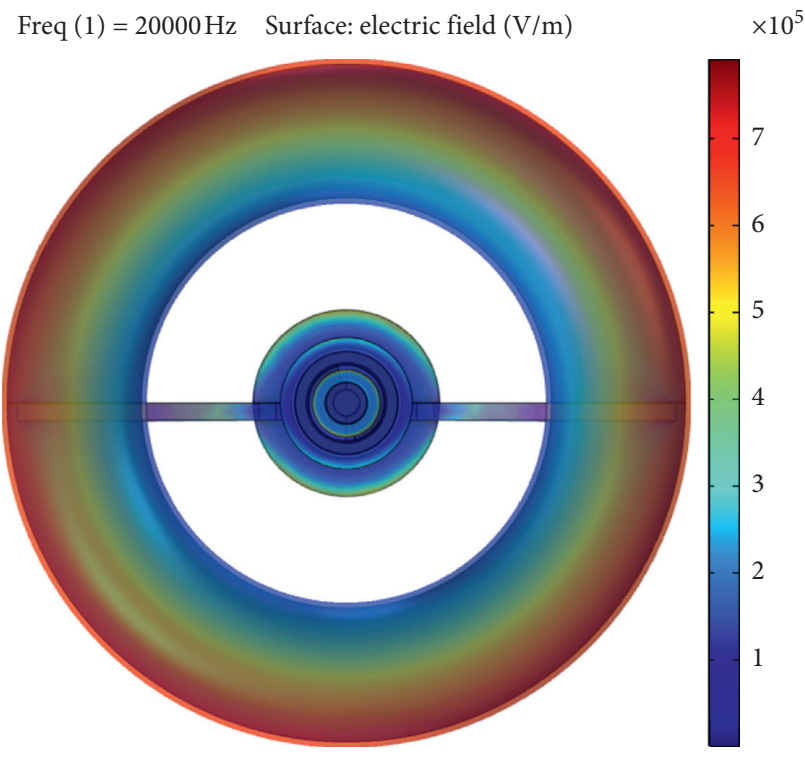

(b)

FIgURE 11: The simulation results of the VLF antenna with the corona ring which has the optimal structural parameters. (a) The electric field distribution at the antenna end. (b) The electric field distribution of the whole part.

corresponding dimensions which are shown in Table 2 within the iteration times of 10 and stay constant after that.

4.2. Optimization through SA. Though it is fast to gain the best parameters, it is doubtful whether the particles get stuck to the local optimal value. Therefore, the SA algorithm was used to test the accuracy of the result in the next section.
The SA algorithm was proposed by Kirkpatrick in 1984 [20]. It is often used in the optimization of electromagnetic devices because of its robustness, simplicity of implementation, and ability to find the global extremum of the objective function [21]. The idea of finding the optimal is that one first finds the melting temperature by starting at an arbitrary temperature, attempting a few hundred moves, and 
determining the fraction of moves. If that fraction is less than a value, the temperature is doubled. When the fraction of moves exceeds the threshold, a sufficient number of movements are taken to completely melt the system, and the cooling process begins. The process is that one decreases the temperature by a constant ratio, running at each temperature until every movable object has been moved a fixed number of times, or some allotted numbers of attempts at that temperature have been taken [20]. The acceptance probability of a trial solution point $X_{i+1}$ from the current solution point $X_{i}$ is stated as follows:

$$
P= \begin{cases}1, & \text { if } f\left(X_{i+1}\right) \leq f\left(X_{i}\right), \\ e^{-\Delta f / T}, & \text { otherwise, }\end{cases}
$$

where $f$ is the objective function, $\Delta f=f\left(X_{i+1}\right)-f\left(X_{i}\right)$, and $T$ is the controlling parameter decreasing slowly during the optimization process as

$$
T_{i+1}=R_{T} T_{i}
$$

where $T_{i}$ is the current temperature and $R_{T}$ is a constant between 0.8 and 0.99 [15]. If the probability $P=1$, it means that the option is accepted. However, if the contrary occurs, there is the probability of the system taking an uphill movement [22].

Though this approach has the great drawbacks that the number of sampling points per variable fixes the resolution on the objective function [23], the algorithm has to sample the objective function in a very high number of points in parameter space in order to work efficiently [24], and the information accumulated in the searched parameter space is not used to guide the generation of new states which shall cause computational inefficiency. The SA was utilized to compensate for the probable drawback of the PSO, and the objective function was not too complex to cause high computational cost. Therefore, the traditional SA was good enough to avoid the probable local minimum and prove the accuracy of the results.

The simulated annealing solver of the optimization tool was utilized to chase for the optimal. The max iteration was specified as 1500, and the other parameters were set to default option including the max function evaluations of $3000^{*}$ number of variables, the stall iterations of $500^{*}$ number of variables, the reannealing interval of 100 , and the initial temperature of 100 . The process is presented in Figure 10. As exhibited in the figure, the SA converges to the optimal value after the iteration times of almost 900 with the result shown in Table 2, which is really close to the ones of PSO. The results show that the performances of the two algorithms are reliable.

\section{Verification through the Simulation}

However, whether the final results are convincing depends on the accuracy of the fitting function too. Therefore, it is indispensable to prove the accuracy of the outcome by simulations.

With the consideration of the practical accuracy of the manufacture, the ring depth was adjusted to $193 \mathrm{~mm}$. The simulation results of the antenna with the grading ring which has the optimal structure parameters are presented in Figure 11. The effect of restraining electric field distortion in the simulation is even better than the expectation. The electric field distribution has strict symmetry, which indicates that the quality of the simulation is satisfactory. Therefore, there is every reason to insist that the result gained by optimization is the optimal parameters within the range illustrated above.

It can be seen from Figure 11 that, by applying the corona ring with the optimal structural parameters, the maximum electric field intensity at the antenna end decreased to $5.27 \times 10^{5} \mathrm{~V} / \mathrm{m}$. When compared to the result of Figure 3, which is $1.36 \times 10^{6} \mathrm{~V} / \mathrm{m}$, the electric field intensity is decreased by additional $61.20 \%$ based on the standard of the medium-sized grading ring. The result illustrates that the method of applying the optimal corona ring is truly effective in reducing the electric field intensity at the antenna and improving the electric field distribution. This shall fundamentally solve the serious problem of temperature rise at the terminal of the VLF antenna.

\section{Conclusion}

Applying the corona ring is an effective method to improve electric field distribution and restrain temperature rise at the terminal of the VLF antenna. As the tube radius increases or as the ring radius decreases, the electric field intensity has a tendency of monotonic decrease while as the ring depth grows, the electric field intensity decreases first and then rebounds. Increase in the tube radius has the most remarkable attribution on decreasing electric field intensity at the antenna end compared to choosing the smaller ring radius and appropriate ring depth. Through applying the constructed relation function to the PSO and SA algorithms, the gained optimal parameters of the corona ring tend to point to almost the same values: ring tube radius of $40 \mathrm{~mm}$, ring radius of $150 \mathrm{~mm}$, and ring depth of $193 \mathrm{~mm}$. The simulation results verify the effectiveness that by using the corona ring with optimized structural parameters, the electric field intensity at the end of the antenna is greatly reduced, and the electric field distribution is further improved.

\section{Data Availability}

All data, models, or codes used to support the findings of this study are available from the corresponding author upon request.

\section{Conflicts of Interest}

The authors declare that they have no conflicts of interest.

\section{References}

[1] K. Mao, X. Li, C. Wei et al., "Numerical analysis of electric field distribution at the terminal of VLF antenna," in Proceedings of the 2019 IEEE 19th International Conference on 
Communication Technology (ICCT), pp. 819-822, Xi'an, China, October 2019.

[2] B. M'Hamdi, M. Teguar, and A. Mekhaldi, "Optimal design of corona ring on HV composite insulator using PSO approach with dynamic population size," IEEE Transactions on Dielectrics and Electrical Insulation, vol. 23, no. 2, pp. 1048-1057, 2016.

[3] R. Turner, "Submarine communication antenna systems," Proceedings of the IRE, vol. 47, no. 5, pp. 735-739, 1959.

[4] B. Benhabiles, P. Lacour, M. Pellet, C. Pichot, and A. Papiernik, "A study of VLF antennas immersed in sea water: theoretical, numerical, and experimental results," IEEE Antennas and Propagation Magazine, vol. 38, no. 5, pp. 19-29, 1996.

[5] J. Chen, D. Su, Y. Liu et al., "Simulation analysis of airborne VLF Wire Antennas based on FEM," in Proceedings of the 2011 International Conference on Electronics, Communications and Control (ICECC), pp. 2276-2279, Ningbo, China, September 2011.

[6] D. E. Hurdsman, P. M. J. Hansen, and W. Rockway, "LF and VLF antenna modeling," in Proceedings of the IEEE Antennas and Propagation Society International Symposium. Digest. Held in conjunction with: USNC/CNC/URSI North American Radio Sci. Meeting (Cat. No.03CH37450), vol. 4, pp. 811-814, Columbus, OH, USA, June 2003.

[7] Y. Liang and Z. Zhang, "Modeling of VLF valley transmitting antenna," in Proceedings of the 2009 3rd IEEE International Symposium on Microwave, Antenna, Propagation and EMC Technologies for Wireless Communications, pp. 48-50, Beijing, China, October 2009.

[8] H. Koons and M. Dazey, "High-power VLF transmitter facility utilizing a balloon lofted antenna," IEEE Transactions on Antennas and Propagation, vol. 31, no. 2, pp. 243-248, 1983.

[9] B. Li, C. Liu, Y. Kang et al., "Optimal design of top-wires insulator grading rings on VLF transmitting antenna based on the method of moments and butterfly mating optimization," High Voltage Apparatus, vol. 56, pp. 94-100+106, 2020.

[10] S. Zhang, Z. Peng, L. Peng et al., "Optimization of corona ring structure for UHV composite insulator using finite element method and PSO algorithm," in Proceedings of the 2013 IEEE International Conference on Solid Dielectrics (ICSD), pp. 210-213, Bologna, Italy, June 2013.

[11] M. R. Aghaebrahimi, R. Shariatinasab, and M. Ghayedi, "Optimal design of grading ring of surge arresters due to electric field distribution," in Proceedings of the 2012 16th IEEE Mediterranean Electrotechnical Conference, pp. 548-550, Yasmine Hammamet, Tunisia, March 2012.

[12] S. Ilhan and A. Ozdemir, " $380 \mathrm{kV}$ corona ring optimization for AC voltages," IEEE Transactions on Dielectrics and Electrical Insulation, vol. 18, no. 2, pp. 408-417, 2011.

[13] R. M. R. Barros, E. G. Da Costa, T. V Ferreira et al., "A new approach for optimal design of corona ring," in Proceedings of the 2016 IEEE International Power Modulator and High Voltage Conference (IPMHVC), pp. 684-687, San Francisco, CA, USA, July 2016.

[14] J. Dong and J. Luo, "Analysis of VLF balloon antenna cable terminal loss and heating-up," Ship Electronic Engineering, vol. 35, pp. 159-161, 2015.

[15] J. Wei and J. Zhang, "Reliability algorithm based on adaptive hybrid particle swarm optimization and simulated annealing algorithm," in Proceedings of the 2017 International Conference on Computer Technology, Electronics and Communication (ICCTEC), pp. 508-512, Dalian, China, December 2017.
[16] M. Merchaoui, A. Sakly, and M. F Mimouni, "Improved fast particle swarm optimization based PV MPPT," in Proceedings of the 2018 9th International Renewable Energy Congress (IREC), pp. 1-7, Hammamet, Tunisia, March 2018.

[17] S. Bashath and A. R. Ismail, "Improved particle swarm optimization by fast simulated annealing algorithm," in Proceedings of the 2019 International Conference of Artificial Intelligence and Information Technology (ICAIIT), pp. 297301, Peking, China, March 2019.

[18] J. Kennedy and R. Eberhart, "Particle swarm optimization," in Proceedings of ICNN'95-International Conference on Neural Networks, vol. 4, pp. 1942-1948, Perth, Australia, November 1995.

[19] Y. Zheng, W. Wang, W. Chen et al., "Research on MPPT of photovoltaic system based on PSO under partial shading condition," in Proceedings of the 2016 35th Chinese Control Conference (CCC), pp. 8654-8659, Chengdu, China, July 2016.

[20] S. Kirkpatrick, "Optimization by simulated annealing: quantitative studies," Journal of Statistical Physics, vol. 34, no. 5-6, pp. 975-986, 1984.

[21] S. Alfonzetti, E. Dilettos, and N. Salerno, "Simulated annealing with restarts for the optimization of electromagnetic devices," IEEE Transactions on Magnetics, vol. 42, no. 4, pp. 1115-1118, 2006.

[22] R. Tang Renyuan, J. Sun Jianzhong, and Y. Cui Xiang, "Optimization of electromagnetic devices by using intelligent simulated annealing algorithm," IEEE Transactions on Magnetics, vol. 34, no. 5, pp. 2992-2995, 1998.

[23] G. Drago, A. Manella, M. Nervi, M. Repetto, and G. Secondo, "A combined strategy for optimization in nonlinear magnetic problems using simulated annealing and search techniques," IEEE Transactions on Magnetics, vol. 28, no. 2, pp. 1541-1544, 1992.

[24] P. Alotto, A. Caiti, G. Molinari, and M. Repetto, "A multiquadrics-based algorithm for the acceleration of simulated annealing optimization procedures," IEEE Transactions on Magnetics, vol. 32, no. 3, pp. 1198-1201, 1996. 